\title{
Indexable signal amplification for multiparametric imaging
}

Paul D. Simonson*, Itzel Valencia, and Sanjay S. Patel

Department of Pathology and Laboratory Medicine, Weill Cornell Medicine, New York, NY

*Corresponding author:

Paul D. Simonson, M.D., Ph.D.

Department of Clinical Pathology and Laboratory Medicine

Weill Cornell Medicine

525 E $68^{\text {th }}$ Street, Starr 702B

New York, NY 10021

pds9003@med.cornell.edu

\section{Acknowledgements}

This work was supported by the Department of Clinical Pathology and Laboratory Medicine at Weill Cornell Medicine. We thank Giorgio Inghirami and Massimo Loda for review of the manuscript and constructive comments prior to submission for publication.

\section{Contributions}

P.D.S. developed the tyramide-oligomer constructs, designed the coverslip adapter, developed staining protocols for antibody and in situ hybridization staining, directed the work, and wrote the paper. I.V. developed staining protocols, carried out the experimental work, and contributed to writing the paper. S.S.P. was engaged in discussion of tyramide-oligomer constructs, developing the staining protocols, and writing the paper. 
bioRxiv preprint doi: https://doi.org/10.1101/2021.06.03.446843; this version posted June 3, 2021. The copyright holder for this preprint (which was not certified by peer review) is the author/funder. All rights reserved. No reuse allowed without permission.

\section{Competing Interests Declaration}

Cornell University has filed a provisional patent application related to work presented here, with P.D.S. and

S.S.P. listed as inventors. 


\section{Indexable signal amplification for multiparametric imaging}

\section{Key Words}

tyramide signal amplification, multiparametric imaging, multiparametric in situ imaging, multicolor imaging, CODEX, barcode, tumor microenvironment, in situ hybridization

\section{Abstract}

Multiparametric imaging allows researchers to measure the expression of many biomarkers simultaneously, allowing detailed characterization of cell microenvironments. One such technique, CODEX, allows fluorescence imaging of $>30$ proteins in a single tissue section. In the commercial CODEX system, primary antibodies are conjugated to DNA barcodes. This modification can result in antibody dysfunction, and development of a custom antibody panel can be very costly and time consuming as trial and error of modified antibodies proceeds. To address these challenges, we developed novel tyramide-conjugated DNA barcodes that can be used with primary antibodies via peroxidaseconjugated secondary antibodies. This approach results in signal amplification and imaging without the need to conjugate primary antibodies. When combined with commercially available barcode-conjugated primary antibodies, we can very quickly develop working antibody panels. We also present methods to perform antibody staining using a commercially available automated tissue stainer and in situ hybridization imaging on a CODEX platform.

\section{Introduction}

Characterization of tumor microenvironments is of great interest for understanding cancer biology and identifying potential targeted therapies ${ }^{1,2}$. While flow cytometry, including CyTOF ${ }^{3}$, allows comprehensive immunoprofiling of cell suspensions, cell-to-cell interactions and other spatial information is lost. Multiparametric tissue imaging, on the other hand, enables characterization of tumor microenvironments with many biomarkers while preserving spatial context ${ }^{4-6}$. Examples of the utility of multiparametric imaging to identify potential targeted disease therapies are multiplying, 
including CTLA4-blockade for classic Hodgkin lymphoma ${ }^{7}$ and hypomethylating agents for subsets of diffuse large B cell lymphoma patients ${ }^{8}$.

Current multiparametric imaging platforms vary by maximum number of concurrent targets, throughput, and fundamental methodologies. High throughput platforms like the Opal/Vectra system (Akoya Biosciences) provide the benefit of signal amplification $n^{5,9}$ but are hindered by low multiplexing capacity (generally six or fewer antibodies) and difficulties associated with spectral overlap of the fluorescent molecules used as reporters. More highly multiplexing approaches, including CO-Detection by indEXing $(C O D E X)^{10,11}$, Digital Spatial Profiling ${ }^{12}$, cyclic immunofluorescence $(\text { CyclF })^{13-16}$, Imaging Mass Cytometry (Fluidigm) ${ }^{17}$, and multiplexed ion beam imaging by time of flight mass spectrometry $(\mathrm{MIBI}-\mathrm{TOF})^{18,19}$, can image more biomarkers but do not allow signal amplification and are costly, low-throughput, and technically- and labor-intensive. Ultivue InSituPlex is a highly multiplexable solution that allows signal amplification of DNA-barcoded antibodies through the use of a proprietary method ${ }^{6,20}$, though the need for primary antibody conjugation to DNA barcodes remains. Immuno-SABER ${ }^{21}$ is also a highly multiplexable method that allows signal amplification via attachment of extended DNA oligomers with several attached fluorophores but, again, primary antibody conjugation to DNA barcodes is required.

Conjugating antibodies to DNA barcodes, necessary for CODEX and other imaging systems, can result in antibody dysfunction. Development of an antibody panel can therefore be very costly and time consuming as trial and error of modified antibodies proceeds. In some cases, a working barcode-conjugated antibody clone might simply not be identified after great time and expense. Even if a working conjugated antibody is produced, some biomarkers are expressed in such low quantities that the imaging intensity might be insufficient for reliable interpretation. Recently Mistry et al. demonstrated an approach for tyramide signal amplification in CODEX ${ }^{22}$ with three amplified marker signals (presumably one for each of the available microscope fluorescence channels), and the imaging was performed after completion of the normal CODEX imaging. This approach, while useful, is limited due to the inability to go beyond the number of available fluorescence channels and the need for removing the sample from the imaging system for additional staining and re-imaging. 
In this report, we present a generalizable method for CODEX imaging with unmodified primary antibodies and signal amplification that uses novel tyramide-conjugated DNA barcodes (tyramide-barcodes). This allows us to use the benefits of tyramide signal amplification, but image the targets in an indexed way using a commercial CODEX imaging system. This approach is flexible, and we also demonstrate that it can be used with in situ hybridization probes. In order to make this system workable, we also developed a staining approach that allows us to perform both tyramide-barcode staining and standard CODEX staining using a commercially available automated tissue stainer, made possible using a custom coverslip holder. This drastically reduces the amount of manual labor required for CODEX staining and has the potential to improve staining reproducibility.

\section{Results}

\section{Staining automation}

In order to integrate tyramide-barcode staining with regular CODEX staining using barcode-conjugated primary antibodies, we determined it was desirable to automate the staining of tissue samples for CODEX imaging. In the commercial version of CODEX, antibody staining is carried out manually using tissue attached to a coverslip. A coverslip is required for the optical imaging, yet commercial automated stainers are generally designed to work with microscope slides. We therefore developed a custom metal coverslip adapter (Fig. S1), which enabled us to stain coverslips on a commercial tissue autostainer. We were then able to adapt the manual CODEX staining protocols to the autostainer, with final fixation of antibodies to the tissue performed manually after removal of the coverslip from the autostainer.

\section{Tyramide-barcode staining is compatible with CODEX imaging}

In the tyramide-barcode staining approach, primary antibody is incubated with tissue, followed by secondary (or secondary followed by tertiary) antibody that is conjugated to peroxidase. The sample is then incubated with tyramidebarcode. These steps are performed in the autostainer and repeated for as many primary antibodies as is desired, after which standard CODEX staining with a cocktail of barcode-conjugated primary antibodies can also be performed (See

Fig. 1 and Methods). Initial attempts used single-stranded tyramide-barcodes in the autostainer; however, these 
experiments consistently failed. Switching to double-stranded tyramide-barcodes resulted in consistently appropriate staining. As proof of principle, we began by imaging one, then two, antigens within a single tissue section (for example, see Fig. S2). We then demonstrated that we could cycle tyramide-barcode imaging on and off, with appropriate clearing between imaging cycles. We did this by imaging a single antigen, $C D 3 e$ in tonsil tissue, stained using a single tyramidebarcode. We imaged the CD3e in two cycles on a CODEX system. In the first cycle, we imaged using complementary strands attached to Cy5 fluorophore. In the next cycle, we imaged using complementary strands attached to Cy3. We saw appropriate clearing and staining for both cycles (see Fig. S3). Importantly, we found it necessary to use nonfluorescent tyramide-conjugated DNA barcodes when incubating with peroxidase in the autostainer; otherwise, bright fluorescence would be present throughout imaging, despite tissue clearing. This finding suggests that in fact a significant amount of tyramide-conjugated double-stranded DNA barcode was attached to the tissue in such a way that some of the DNA could not be dissociated and washed away, under the tested conditions.

Given these successes, we extended the tyramide-barcode staining technique to imaging using in situ hybridization probes for detection of RNA. Epstein-Barr-virus-encoded small RNAs (EBERs) are noncoding RNAs that are expressed abundantly in EBV-infected cells, and in situ hybridization probes are commonly used to detect them in clinical laboratories. These probes usually have an antigen attached (i.e., FITC), and anti-FITC primary antibodies, followed by secondary, then tertiary peroxidase-conjugated antibodies are used for detection using a chromogenic substrate (catalyzed by peroxidase). We performed tyramide-barcode staining by simply replacing the chromogenic substrate with tyramide-barcode, and we tested the system using a sample of classic Hodgkin lymphoma tissue wherein the Hodgkin cells were known to be infected with EBV. Our system clearly highlighted Hodgkin cells (Fig. S4) and was appropriately negative for negative controls. These findings demonstrate the flexibility of the tyramide-barcode approach, beyond just protein detection via primary antibodies, for CODEX imaging.

We then demonstrated tyramide-barcode staining with multiple primary antibodies. This required multiple staining cycles, one for each unique primary antibody, with inactivation of peroxidase prior to incubation with each 
subsequent primary antibody. Staining lymph node tissue with four primary antibodies (CD3e, CD4, CD10, and ICOS) in this manner demonstrated appropriately localized staining (see Fig. S5).

\section{Integration with standard CODEX antibodies}

We next incorporated tyramide-barcode staining with multiple primary antibodies into a complete CODEX workflow so that we could stain some markers with tyramide-barcodes and some with commercially available barcodeconjugated primary antibodies. We successfully performed all staining steps in an autostainer. We found it important to minimize the stripping of antibodies in the tyramide staining steps to maximize the signal seen by conjugated primary antibodies. Example images demonstrating the results of the integrated staining protocol are seen in Fig. 2.

\section{Rapid antibody panel development for classic Hodgkin lymphoma}

As an example of the use of the full method, we imaged classic Hodgkin lymphoma using a custom CODEX antibody panel that was primarily comprised of commercially available barcode-conjugated primary antibodies, but also included antibodies for two additional markers, CD30 and MUM1, for which commercial barcode-conjugated antibodies are not available. Prior experience in CD30 antibody conjugation with barcodes had been less than satisfactory. By staining CD30 and MUM1 antigens using tyramide-barcodes, and other antigens using commercial barcode-conjugated antibodies, we were able to image all the antigens on a CODEX system with very little panel development effort and no custom antibody conjugation. DAPI and eosin fluorescence imaging was performed using the CODEX instrument at the end of the imaging run for virtual H\&E imaging ${ }^{23}$ for morphologic comparison with CODEX images in the same tissue section (see Fig. 3).

\section{Discussion}

Standard CODEX imaging makes use of primary antibodies conjugated with DNA oligomers (barcodes), which are in turn hybridized with complementary fluorophore-conjugated DNA strands ${ }^{11}$. Fluorescence imaging is performed in cycles, with only a few complementary strands imaged per cycle so as to avoid the problem of spectral overlap that would be had if many fluorophores were used ${ }^{10,11}$. Cycling continues until all of the antigen types, indexed by barcodes, 
have been imaged (see stage 2 in Fig. 1). In the normal situation, there is no signal amplification since the number of barcodes is proportional to the number of bound primary antibodies. Thus, this approach is limited.

In this work, we have developed new reagents, tyramide-barcodes, that can be used to stain tissue using unmodified primary antibodies and achieve signal amplification. Tyramide-barcodes are flexible and can in theory be used in any peroxidase-based signal amplification system, including in in situ hybridization imaging approaches (see Fig. S4). Use of barcodes means that in theory a large number of signals can be amplified.

Besides signal amplification, perhaps the greatest benefit of this new approach is the lack of need for primary antibody conjugation with DNA barcodes. This approach is compatible with any primary antibody, including those supplied in solutions with carrier protein (e.g. bovine serum albumin), which normally must be absent when conjugating antibodies to DNA barcodes or lanthanide metals (for Imaging Mass Cytometry). Thus, it is possible to use virtually all commercially available antibodies used for immunohistochemistry, with no need for additional purification. Moreover, primary antibodies previously validated by a given lab should be compatible, obviating the need to purchase new reagents and enabling the lab members to capitalize on their prior expertise with a given antibody clone. That experience can help direct optimization of the staining protocol, which is in fact significant given that the staining conditions (time, concentration, etc.) can be customized for each antibody. In our own experience, development of a custom CODEX antibody panel can be significantly hindered by just a few troublesome biomarkers for which a working barcode-conjugated antibody clone is difficult to find. By instead using the tyramide-barcode staining approach, as we did for CD30 staining in classic Hodgkin lymphoma, experiments can go forward without an inordinate amount of time and money spent on the few troublesome biomarkers. The net result is increased efficiency at a reduced cost.

There are limitations to the tyramide-barcode staining approach. One potential limitation could be steric blocking by tyramide-barcodes of antibodies to antigens in later staining steps. For example, in T cells, if CD3e staining signal is amplified in an early staining step, it is possible that CD4 staining in a later step would be reduced due to the CD3associated barcodes blocking the binding of the CD4 antibodies. We note, however, that in Opal/Vectra imaging, this potential steric hindrance is not generally observed to be an obstacle. Another potential limitation is that the ability to 
quantify the amount of antigen present is reduced since the signal is no longer linearly proportional to the amount of bound primary antibody. The approach is limited by the amount of time the staining takes to complete since for each primary antibody used for amplification, the process can take 1-2 hours to complete (hence, we normally perform the automated tissue staining overnight). Excessive peroxidase blocking and/or removal of antibodies between tyramidebarcode amplification cycles could also lead to increased tissue deterioration for downstream biomarker staining. Hence, limiting the number of amplified biomarker signals is still considered advantageous in that it saves staining time and reduces possible tissue deterioration.

In summary, we have developed tyramide-conjugated DNA barcodes that can be used with unmodified primary antibodies in a CODEX imaging system. These enable amplification of the staining signal and imaging of biomarkers for which barcode-conjugated primary antibodies are difficult to generate. We have developed a custom coverslip holder and associated automated CODEX staining workflow, which also allows combining both tyramide-conjugated barcodes and standard barcode-conjugated primary antibodies in a single workflow. Future work will include further refinement of the staining protocols, use of additional fluorophores, and further incorporation of in situ hybridization probes. We will apply the approach to many more imaging applications as we seek to further understand cellular microenvironments and their potential utility for predicting clinical outcomes and response to therapy.

\section{Methods}

Additional methods can be found in the Supplementary Information.

\section{Construction of tyramide-barcodes}

DNA barcode sequences were selected from a list of oligomers that were previously reported as successfully used in CODEX imaging ${ }^{11}$. For one construct, we created the following custom-conjugated oligomer ( $5^{\prime}$ to $3^{\prime}$, custom order to GeneLink, Elmhurst, New York):

/tyramide/C10/CAAGGAACTACCGA 
where $\mathrm{C} 10$ is a linker consisting of 10 carbon atoms. Tyramide was attached to the linker via an NHS ester linkage. Complementary DNA oligomers were also made, with and without attached fluorophores at the $5^{\prime}$ end. Stock solutions were made by diluting oligomer to $1 \mathrm{mg} / \mathrm{ml}$ in TE $\mathrm{pH} 8.0$ buffer and stored at $4^{\circ} \mathrm{C}$ or less.

\section{Autostainer coverslip holders}

The coverslip holder was designed using FreeCAD software (see Fig. S1) and manufactured using CNC machining (ProtoLabs, Maple Plain, Minnesota). The long edges were outlined by a hydrophobic pen (Hydrophobic Barrier PAP Pen, Vector Laboratories) to reduce the chances of leakage in the instrument, though significant leakage has not yet been observed. Before placing a coverslip on the coverslip holder, a small droplet of PBS buffer is placed to help the coverslip adhere. The coverslip holder is washed with water and ethanol between uses.

\section{Combined tyramide-barcode and barcode-conjugated primary antibody staining}

For the imaging seen in Fig. 2, de-identified tonsil tissue was cut into 4-micron-thick sections and placed on charged coverslips. Using a Leica Bond RX automated stainer and our custom coverslip holder, the tissue was deparaffinized, followed by antigen retrieval (Leica ER2 solution, 20 minutes). The protocol then proceeded as follows with Bond Wash Solution (Leica) used for washing: Leica peroxidase blocking solution (5 minutes), wash 3x, mouse CD4 antibody diluted in Abcam antibody diluent (30 minutes), wash 3x for 2 minutes each, Leica rabbit anti-mouse antibody (8 minutes), wash $3 x$ for 2 minutes each, Leica anti-rabbit antibody conjugated with HRP (8 minutes), wash 3x for 2 minutes each, double-stranded tyramide-barcode \#76 ${ }^{11}$ (30 minutes), wash (5 minutes), Leica peroxidase blocking solution (5 minutes), wash 3x, CD163 diluted in Abcam antibody diluent (30 minutes), wash 3x for 2 minutes each, Leica rabbit anti-mouse antibody (8 minutes), wash $3 x$ for 2 minutes each, Leica anti-rabbit antibody conjugated with HRP (8 minutes), wash $3 x$ for 2 minutes each, double-stranded tyramide-barcode \#79 11 (30 minutes), de-ionized water $2 x$ for 10 minutes each, Akoya hydration buffer $2 x$ for 2 minutes each, Akoya staining buffer ( 25 minutes), conjugated primary antibodies cocktail (Akoya CD3e 1:100 and Akoya CD20 1:200 in Abcam Antibody Diluent) 2x for 90 minutes each, wash.

The coverslip was then washed 2x with Akoya Hydration Buffer for 2 minutes each before being placed in 1.6\% formalin in Akoya Hydration Buffer for 10 minutes. The coverslip was then washed 3x in PBS before being placed in cold 
methanol for 5 minutes. The coverslip was then washed 3x before being placed briefly in Akoya Storage Buffer. Imaging then proceeded as usual for CODEX imaging using the protocols outlined in the Akoya CODEX manual (revision C), but with solutions of $400 \mathrm{nM}$ Cy5-conjugated complementary barcode used as appropriate for the tyramide-barcode stained markers (CD4 and CD163). At the conclusion of CODEX imaging, we used a weak solution of DAPI and eosin to acquire fluorescence images to generate virtual H\&E images ${ }^{23}$.

\section{Antibody staining of classic Hodgkin lymphoma}

Staining of classic Hodgkin lymphoma (Fig. 3) proceeded as outlined above, with a tyramide-barcode staining cycle performed for CD30 primary antibody, and a tyramide-barcode staining cycle performed for MUM1 primary antibody. However, in this case the tissue was incubated with the barcode-conjugated primary antibodies cocktail in a hydration chamber at $4^{\circ} \mathrm{C}$ overnight rather than inside the autostainer at room temperature for two 90 minute cycles.

\section{Imaging}

A commercial Akoya CODEX instrument and a Keyence BZ-X800 microscope with a 20x Nikon PlanApo NA 0.75 objective were used to clear tissue and stain with complementary fluorescent barcodes. The standard Akoya CODEX protocols were followed (Akoya CODEX manual, revision B or revision C). For tyramide-barcode staining, we replaced the Akoya barcodes in the reporter plate solutions with our custom complementary barcodes, with a final concentration of $\sim 400 \mathrm{nM}$ for each. Post-acquisition image processing was performed using the Akoya CODEX Processor software, and inspection of results was performed using ImageJ with the Akoya CODEX MAV plugin.

\section{References}

1. Taube, J. M. et al. Implications of the tumor immune microenvironment for staging and therapeutics. Modern Pathology 31, 214-234 (2018).

2. Rozenblatt-Rosen, O. et al. The Human Tumor Atlas Network: Charting Tumor Transitions across Space and Time at Single-Cell Resolution. Cell 181, 236-249 (2020). 
3. Kay, A. W., Strauss-Albee, D. M. \& Blish, C. A. Application of Mass Cytometry (CyTOF) for Functional and Phenotypic Analysis of Natural Killer Cells. Methods Mol Biol 1441, 13-26 (2016).

4. Patel, S. S. \& Rodig, S. J. Overview of Tissue Imaging Methods. in Biomarkers for Immunotherapy of Cancer: Methods and Protocols (eds. Thurin, M., Cesano, A. \& Marincola, F. M.) 455-465 (Springer, 2020). doi:10.1007/978-1-49399773-2_21.

5. Stack, E. C., Wang, C., Roman, K. A. \& Hoyt, C. C. Multiplexed immunohistochemistry, imaging, and quantitation: A review, with an assessment of Tyramide signal amplification, multispectral imaging and multiplex analysis. Methods 70, 46-58 (2014).

6. Tan, W. C. C. et al. Overview of multiplex immunohistochemistry/immunofluorescence techniques in the era of cancer immunotherapy. Cancer Communications 40, 135-153 (2020).

7. Patel, S. S. et al. The microenvironmental niche in classic Hodgkin lymphoma is enriched for CTLA-4-positive T cells that are PD-1-negative. Blood 134, 2059-2069 (2019).

8. Kotlov, N. et al. Clinical and biological subtypes of B-cell lymphoma revealed by microenvironmental signatures. Cancer Discov (2021) doi:10.1158/2159-8290.CD-20-0839.

9. Pugia, M., PHILIP, J., Marfurt, K. \& FOLTZ, M. Rare molecule signal amplification. (2015).

10. Goltsev, Y. et al. Deep Profiling of Mouse Splenic Architecture with CODEX Multiplexed Imaging. Cell 174, 968981.e15 (2018).

11. Schürch, C. M. et al. Coordinated Cellular Neighborhoods Orchestrate Antitumoral Immunity at the Colorectal Cancer Invasive Front. Cell 182, 1341-1359.e19 (2020).

12. Merritt, C. R. et al. Multiplex digital spatial profiling of proteins and RNA in fixed tissue. Nature Biotechnology 38, 586-599 (2020).

13. Smith, E. A. \& Hodges, H. C. The spatial and genomic hierarchy of tumor ecosystems revealed by single-cell technologies. Trends Cancer 5, 411-425 (2019).

14. Lin, J.-R., Fallahi-Sichani, M. \& Sorger, P. K. Highly multiplexed imaging of single cells using a high-throughput cyclic immunofluorescence method. Nature Communications 6, 8390 (2015). 
15. Lin, J.-R., Fallahi-Sichani, M., Chen, J.-Y. \& Sorger, P. K. Cyclic Immunofluorescence (CyclF), A Highly Multiplexed Method for Single-cell Imaging. Curr Protoc Chem Biol 8, 251-264 (2016).

16. Lin, J.-R. et al. Highly multiplexed immunofluorescence imaging of human tissues and tumors using t-CyClF and conventional optical microscopes. eLife 7, e31657 (2018).

17. Giesen, C. et al. Highly multiplexed imaging of tumor tissues with subcellular resolution by mass cytometry. Nature Methods 11, 417-422 (2014).

18. Keren, L. et al. MIBI-TOF: A multiplexed imaging platform relates cellular phenotypes and tissue structure. Science Advances 5, eaax5851 (2019).

19. Angelo, M. et al. Multiplexed ion beam imaging of human breast tumors. Nature Medicine 20, 436-442 (2014).

20. Manesse, M., Patel, K. K., Bobrow, M. \& Downing, S. R. The InSituPlex ${ }^{\circledR}$ Staining Method for Multiplexed Immunofluorescence Cell Phenotyping and Spatial Profiling of Tumor FFPE Samples. in Biomarkers for Immunotherapy of Cancer: Methods and Protocols (eds. Thurin, M., Cesano, A. \& Marincola, F. M.) 585-592 (Springer, 2020). doi:10.1007/978-1-4939-9773-2_26.

21. Saka, S. K. et al. Immuno-SABER enables highly multiplexed and amplified protein imaging in tissues. Nat Biotechnol 37, 1080-1090 (2019).

22. Bobrow, M. N., Harris, T. D., Shaughnessy, K. J. \& Litt, G. J. Catalyzed reporter deposition, a novel method of signal amplification. Application to immunoassays. J Immunol Methods 125, 279-285 (1989).

23. Simonson, P. D., Ren, X. \& Fromm, J. R. Creating virtual H\&amp;E images using samples imaged on a commercial CODEX platform. medRxiv 2021.02.05.21249150 (2021) doi:10.1101/2021.02.05.21249150. 


\section{OVERVIEW OF IMAGING USING TYRAMIDE-BARCODES}

\section{Cyclical tyramide-barcode staining and barcode-conjugated antibody staining}

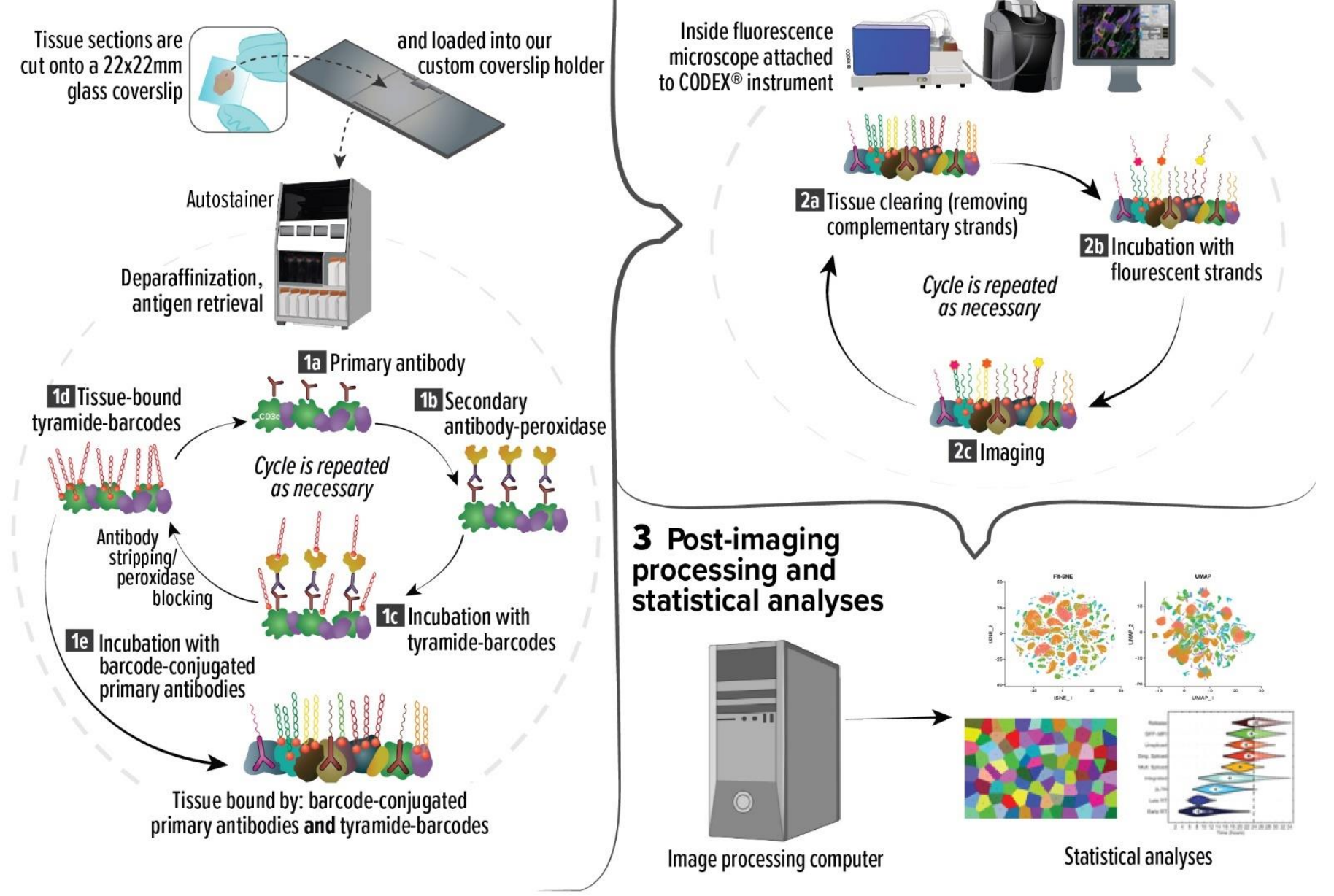

Figure 1. Multiparametric imaging with tyramide-barcodes and barcode-conjugated antibodies. The process can be divided into three stages: (1) tissue staining with tyramide-barcodes and barcode-conjugated antibodies, (2) cyclical fluorescence imaging, and (3) image processing and statistical analysis. In stage 1, tissue is cut into thin sections and placed on microscope coverslips. The coverslips are then loaded onto our custom coverslip holders, which allow processing in a tissue autostainer. Paraffin-embedded tissue is deparaffinized, after which antigen retrieval is performed. Cyclical tyramide-barcode staining is then repeated (steps $1 \mathrm{a}$ to $1 \mathrm{~d}$ ) for as many primary antibodies as desired for signal amplification and/or unmodified primary antibody use. In step 1a, the tissue is incubated with an unmodified primary antibody. In step $1 b$, the tissue is incubated with peroxidase-conjugated secondary antibodies. In step 1c, the peroxidase (attached to the secondary antibodies) enzymatically activates the tyramide, and tyramidebarcodes bind to the tissue in close proximity to the peroxidase. The peroxidase is then permanently inactivated and/or the antibodies are removed, and the cycle can begin again at step 1a for a different primary antibody, or, if there are no more primary antibodies for signal amplification, staining proceeds to step 1e. In step 1e, the tissue is incubated with a cocktail of many barcode-conjugated primary antibodies. The tissue is lightly fixed to lock antibodies into place, and the process then moves on to stage 2. In step 2a, "tissue clearing" is performed, in which double-stranded DNA barcodes are dissociated, and the non-covalently attached strands are washed away. In $2 b$, one to three unique single-stranded 
DNA barcodes are incubated with the tissue, each one conjugated to one of three different fluorophores and complementary to one of the tissue-attached barcodes. After incubation, the tissue is imaged by fluorescence imaging, step2c. By imaging only a small subset of the barcodes in each cycle, high quality imaging of each target antigen can be achieved without significant spectral overlap of fluorescence signals. Steps $2 a-2 c$ are repeated until all of the antigens have been imaged. Stage 3 then commences, comprising post-imaging image alignment, stitching, and segmentation, followed additional study-specific statistical analyses. 

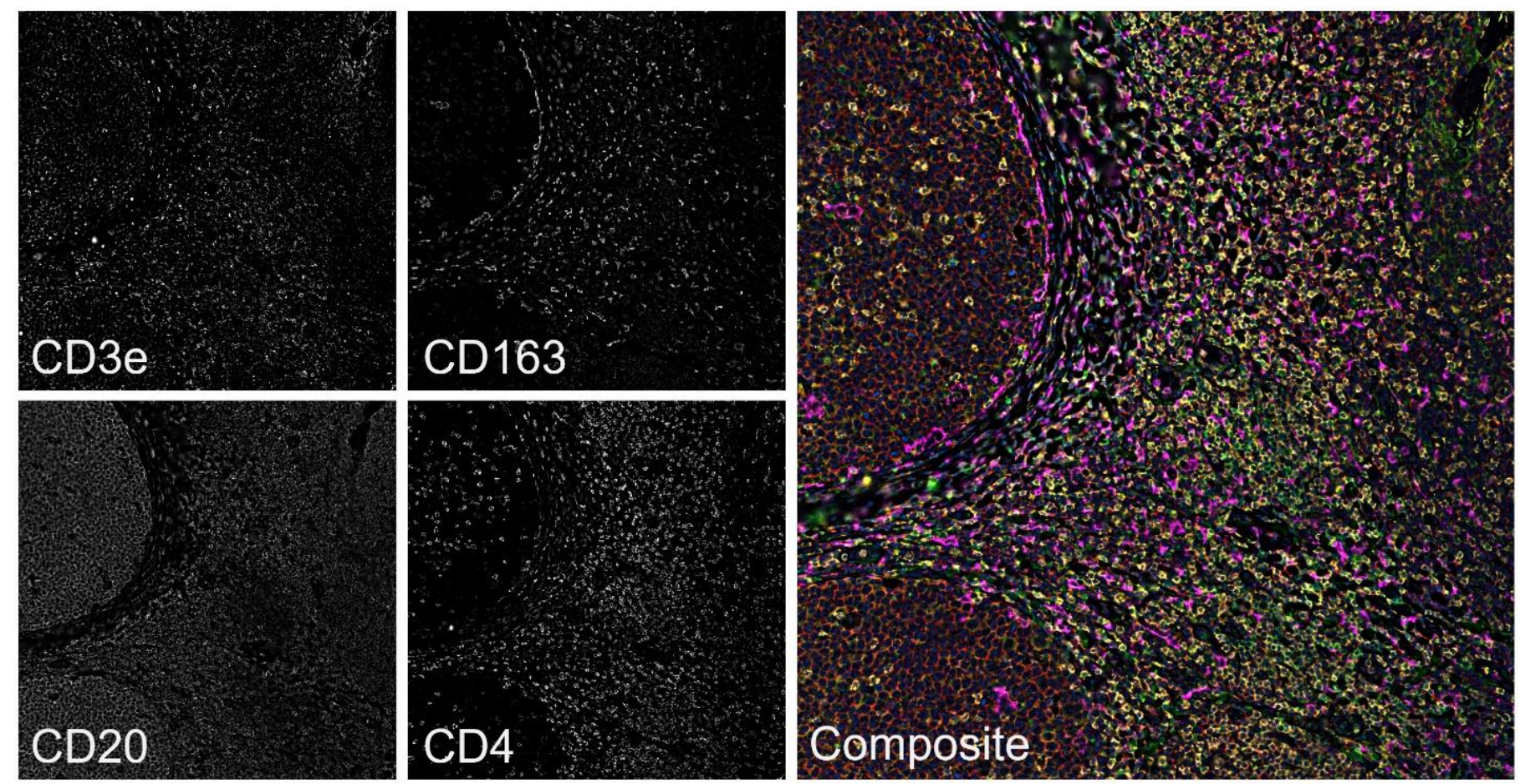

Figure 2. Combined tyramide-barcode and conjugated primary antibody staining in tonsil. CD3e and CD20 were stained using commercial barcode-conjugated primary antibodies, and CD4 and CD163 were stained using tyramidebarcodes. The composite image is colored as follows: $C D 20=$ red, $C D 4=$ yellow, $C D 3 e=$ green, and $C D 163=$ magenta . The Keyence BZ-X800 microscope used 100\% excitation intensity, "high resolution" imaging, and a Nikon 20x PlanApo lambda 0.75 NA objective. Background subtraction and image stitching were performed using Akoya's CODEX Processor software. 


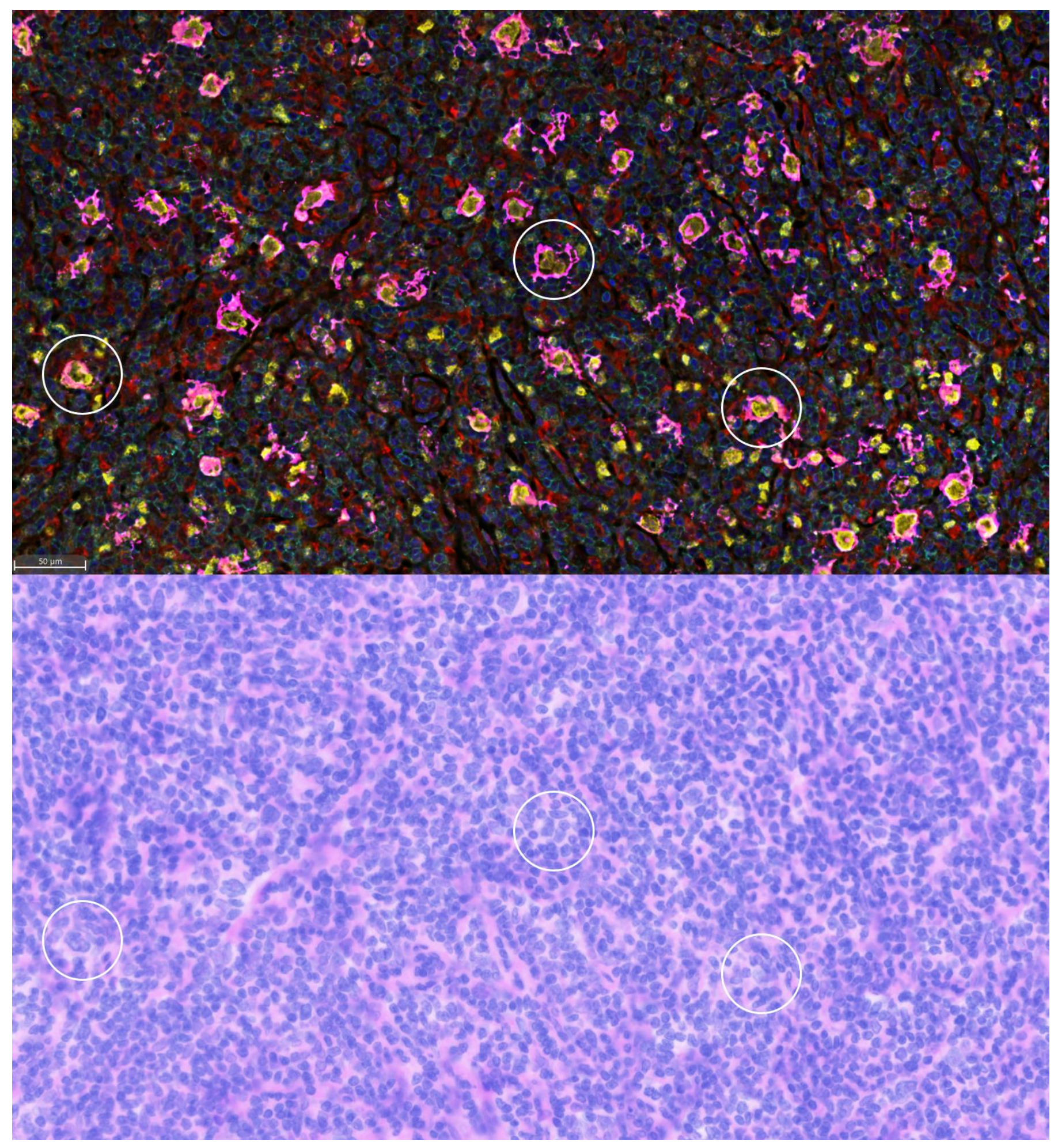

Figure 3. Classic Hodgkin lymphoma stained using a combination of tyramide-barcodes and barcode-conjugated primary antibodies. CD30 and MUM1 staining was performed using the tyramide-barcodes, which stains the cell membranes and nuclei of Hodgkin cells (a few examples are circled), respectively (scattered T cells also express MUM1). Other antigens were stained using commercially available barcode-conjugated primary antibodies. $\mathrm{DAPI}=\mathrm{blue}, \mathrm{CD} 30=$ magenta, MUM1 = yellow, CD68 = red, CD3e = aqua. Not shown: CD4, CD8, CD11c, CD20, CD45RO, CD107a, and Ki67. Virtual H\&E staining (bottom) was generated using DAPI and eosin fluorescence images as described previously ${ }^{23}$. 